\title{
Effect of reentrant cone geometry on energy transport in intense laser-plasma interactions
}

\author{
K. L. Lancaster, ${ }^{1}$ M. Sherlock, ${ }^{1}$ J. S. Green, ${ }^{1,2}$ C. D. Gregory, ${ }^{3}$ P. Hakel, ${ }^{4}$ K. U. Akli, ${ }^{5}$ F. N. Beg, ${ }^{6}$ S. N. Chen, ${ }^{6}$
}

R. R. Freeman, ${ }^{7}$ H. Habara, ${ }^{8}$ R. Heathcote, ${ }^{1}$ D. S. Hey, ${ }^{5}$ K. Highbarger, ${ }^{7}$ M. H. Key, ${ }^{9}$ R. Kodama, ${ }^{10,8}$ K. Krushelnick, ${ }^{11}$ H. Nakamura, ${ }^{10}$ M. Nakatsutsumi, ${ }^{10}$ J. Pasley, ${ }^{11}{ }^{2}$ R. B. Stephens, ${ }^{5}$ M. Storm, ${ }^{13}$ M. Tampo, ${ }^{10}$ W. Theobald, ${ }^{13}$ L. Van Woerkom, ${ }^{7}$ R. L. Weber, ${ }^{7}$ M. S. Wei, ${ }^{6}$ N. C. Woolsey, ${ }^{12}$ T. Yabuuchi, ${ }^{6}$ and P. A. Norreys ${ }^{1,2}$

${ }^{1}$ STFC Rutherford Appleton Laboratory, Chilton, Oxon OX11 0QX, United Kingdom

${ }^{2}$ Blackett Laboratory, Imperial College London, Prince Consort Road, London SW7 2BZ, United Kingdom

${ }^{3}$ LULI, Ecole Polytechnique, Palaiseau Cedex F91128, France

${ }^{4}$ Department of Physics, University of Nevada, Reno, Nevada 89557, USA

${ }^{5}$ General Atomics, P.O. Box 86508, San Diego, California 92186-5608, USA

${ }^{6}$ University of California-San Diego, 9500 Gilman Drive 0411, La Jolla, California 92093-0411, USA

${ }^{7}$ Department of Physics, Ohio State University, Columbus, Ohio 43210-1117, USA

${ }^{8}$ Institute of Laser Engineering, Osaka University, Suita, 565-0871 Osaka, Japan

${ }^{9}$ Lawrence Livermore National Laboratory, P.O. Box 808, Livermore, California 94550, USA

${ }^{10}$ Graduate School of Engineering, Osaka University, Suita, 565-0871 Osaka, Japan

${ }^{11}$ University of Michigan, Ann Arbour, Michigan 48109-2099, USA

${ }^{12}$ Department of Physics, University of York, Heslington, York YO10 5DD, United Kingdom

${ }^{13}$ Laboratory of Laser Energetics, University of Rochester, 250 East River Road, Rochester, New York 14623, USA

(Received 3 February 2009; published 22 October 2009)

\begin{abstract}
The energy transport in cone-guided low- $Z$ targets has been studied for laser intensities on target of 2.5 $\times 10^{20} \mathrm{~W} \mathrm{~cm}^{-2}$. Extreme ultraviolet (XUV) imaging and transverse optical shadowgraphy of the rear surfaces of slab and cone-slab targets show that the cone geometry strongly influences the observed transport patterns. The XUV intensity showed an average spot size of $65 \pm 10 \mu \mathrm{m}$ for slab targets. The cone slabs showed a reduced spot size of $44 \pm 10 \mu \mathrm{m}$. The shadowgraphy for the aforementioned shots demonstrate the same behavior. The transverse size of the expansion pattern was $357 \pm 32 \mu \mathrm{m}$ for the slabs and reduced to $210 \pm 30 \mu \mathrm{m}$. A transport model was constructed which showed that the change in transport pattern is due to suppression of refluxing electrons in the material surrounding the cone.
\end{abstract}

DOI: 10.1103/PhysRevE.80.045401

PACS number(s): 52.38.Kd, 52.57.Kk

The cone-guided fast ignition inertial fusion concept uses a hollow gold cone to keep a channel clear from plasma generated in the compression of a deuterium-tritium fuelfilled capsule to high density. At peak compression, a PW laser pulse is then focused into the cone tip and generates copious numbers of hot electrons that travel into the dense fuel and deposit their energy. Ideally the electron beam must be collimated so that all the useful energy can reach the dense core. It is therefore imperative that a method of controlling electron beam divergence is found.

Previously, it has been shown that, using cone-fiber geometry, it is possible to guide and collimate $\mathrm{MeV}$ electrons along a thin carbon fiber plasma, thereby generating (potentially) gigabar plasma pressures for equation of state studies $[1,2]$. The success of these cone-guided experiments has prompted a theoretical study into the effects of the target geometry [3]. Sentoku et al. [4], for example, used a threedimensional particle-in-cell code and showed that the laser light can be focused to a greater intensity provided that the tip width is the same size as the laser focal spot. The resulting hot electrons converge to the tip due to surface electron flow guided by magnetic and electric fields.

Initial experiments to test this modeling have provided conflicting results. On the one hand, Key et al. [5] showed that, by irradiating the edge of a thick $\mathrm{Cu}$-doped $\mathrm{Al}$ slab with and without an attached hollow $\mathrm{Au}$ cone, there was a lateral spreading of the fast electron beam (as measured by imaging a $\mathrm{Cu} k \alpha$ emission region) in the case of cone-attached geom- etry. On the other hand, Kodama et al. [6] showed that, by irradiating a solid $200-\mu \mathrm{m}$-thick Al slab and a solid cone ( $30^{\circ}$ full angle) attached to $10 \mu \mathrm{m}$ foil, the optical thermal emission (which is directly proportional to the black-body temperature) increased by more than a factor of three in the case of the solid cone slab.

Here we employ targets to show that reentrant cone geometry strongly influences the observed transport pattern in absence of electron refluxing. It is thought that the extra material surrounding the cone suppresses refluxing of the hot electrons inside the target, thus reducing the heated area at the back surface. This experiment is highly relevant to fast ignition since refluxing is not important for transport in that case. A two-dimensional (2D) refluxing model reproduced the size and temperature of the heated region. In the case of these targets, there was no need to invoke "guiding" in order to explain these results.

In this Rapid Communication, the targets were irradiated with petawatt laser pulses from the Vulcan laser facility [7]. The targets were designed to eliminate the vacuum region on the outside of the cone. This is more similar to the intended situation in fast ignition inertial fusion where plasma formed on the outside of the cone supplies the return current, allowing the fast electron beam to more freely propagate.

Diagnosing the physics of the laser interaction inside of the cone is difficult due to the closed geometry. To overcome this limitation, a comparison of energy transport from a cone-slab geometry to planar slab geometry was made, as 

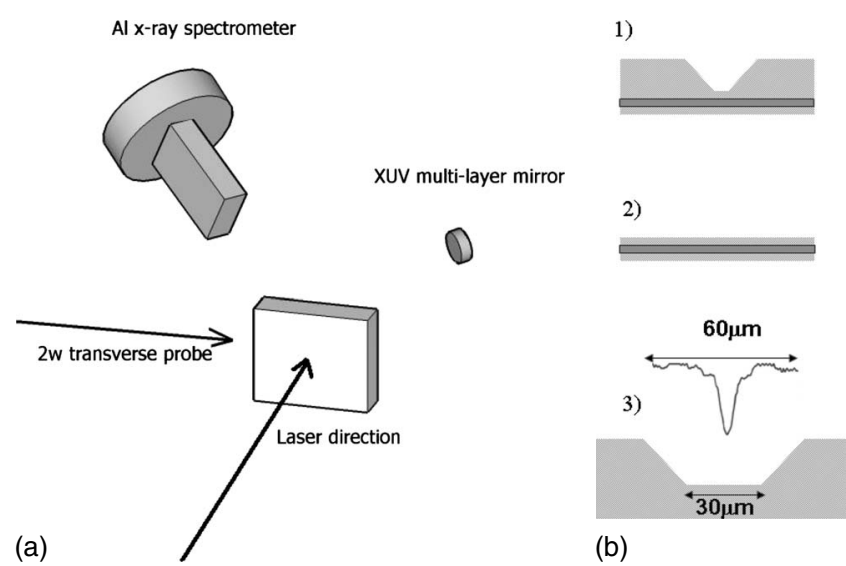

(b)

FIG. 1. Figure (a) shows the layout of the experiment. Figure (b) shows (1) the cone-slab geometry and target, (2) the planar slab geometry, and (3) the focal spot width compared to the size of the cone tip. Note that although the central spot is $7 \mu \mathrm{m}$ there is substantial energy in the wings out to $60 \mu \mathrm{m}$. The cones were $40^{\circ}$ full opening angle.

illustrated in Fig. 1(a). The diagnostics included extreme ultraviolet (XUV) imaging of the rear surface of irradiated targets, transverse shadowgraphy of the interaction, and $\mathrm{Al}$ $\mathrm{X}$-ray spectroscopy of a buried signature layer.

The Vulcan petawatt laser delivered up to $300 \mathrm{~J}$ of $\lambda$ $=1.05 \mu \mathrm{m}$ light on to target in a pulse duration of $700 \mathrm{fs}$. The laser was focused on to target using an $F / 3$ off axis parabolic mirror to a spot size of $7 \mu \mathrm{m}$ diameter. Approximately $20 \%$ of the energy was contained within the central focal spot [8] giving peak intensities up to 2.5 $\times 10^{20} \mathrm{~W} \mathrm{~cm}^{-2}$. The ASE of the laser was $5 \times 10^{-8}, 1.5 \mathrm{~ns}$ ahead of the interaction pulse [9]. The laser was incident on to target at an angle of $0^{\circ}$ and for the cone geometry the laser was incident normally to the tip surface. Buried layer targets consisted of $4 \mu \mathrm{m} \mathrm{CH}, 0.2 \mu \mathrm{m} \mathrm{Al}$, and $4 \mu \mathrm{m} \mathrm{CH}$. A number of targets had $\mathrm{CH}$ cones of $40^{\circ}$ total opening angle attached to the front surface. The cone tip width was $30 \mu \mathrm{m}$, i.e., much larger than the laser focal spot size of $7 \mu \mathrm{m}$. The thickness of the interaction region in both target types were identical $(8 \mu \mathrm{m})$.

A CsAP conically curved x-ray crystal spectrometer [10] was used to record X-ray spectra from the heated Al layer. The spectrometer operated in the wavelength range 6.2-8.4 $\AA$. The center of the crystal and the imaging plane were placed at distances of 12.5 and $25 \mathrm{~cm}$ from the interaction point, respectively. Fujifilm BAS-MS imaging plates were used to record the spectra as this has a approximately flat spectral response over this range [11]. The image plates were read using a Fuji Film BAS 180II reader. The spectrometer had a spectral resolution $>\lambda / \Delta \lambda$ of 1500 .

The heated region at the rear surface was imaged using XUV radiation emitted at 68 and $256 \mathrm{eV}$. The radiation was focused using spherical multilayer mirror onto a Princeton Instruments 16-bit charge coupled device (CCD) camera. The magnification was 12 and the spatial resolution was $10 \mu \mathrm{m}$.

The expansion on the front and back surfaces of the target was diagnosed using a transverse optical probe. The

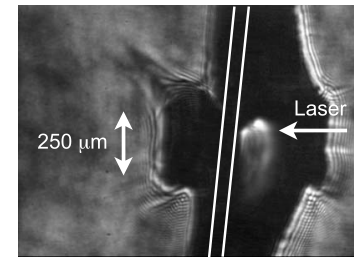

(a)

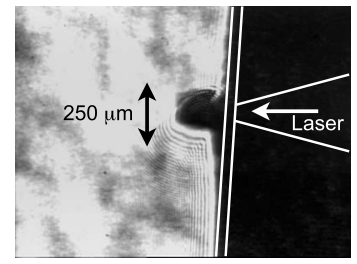

(b)
FIG. 2. Shadowgrams of $4 \mu \mathrm{m} / 0.2 \mu \mathrm{m} / 4 \mu \mathrm{m} \mathrm{CH} / \mathrm{Al} / \mathrm{CH}$ slab targets without (a) and with (b) a plastic guide cone attached. The shadowgrams were taken $400 \mathrm{ps}$ after the main interaction. The original target position is marked on each shadowgram.

$1.054 \mu \mathrm{m}$ light was frequency doubled to $527 \mathrm{~nm}$ using a potassium dihydrogen phosphate (KDP) crystal. The pulse length of the probe and main interaction beam were identical and synchronization of the beams was performed using an optical streak camera.

The shadowgraphy images were recorded at 400 ps after the interaction using an eight-bit CCD camera connected to a personal computer via image acquisition software. The resolution of the probe system was found to be $\sim 12 \mu \mathrm{m}$, the magnification was 5 , and the $f$ number was 12 . Interferograms were also taken, using a Normarski interferometer and a 16 bit Andor technology CCD camera. The dark regions in the images represent plasma at a density higher than a minimum value called the "cut-off" density. Analysis of the interferograms show a cutoff density of $5 \times 10^{19} \mathrm{~cm}^{-1}$.

Shadowgraphs of the slab and cone-slab interactions are presented in Figs. 2(a) and 2(b). The shadowgraph of slab without cone geometry shows regular expansion pattern of transverse size $357 \pm 30 \mu \mathrm{m}$. The shadowgraph of cone-slab geometry shows a smaller transverse region of expansion of size (average) $210 \pm 30 \mu \mathrm{m}$ although the longitudinal extent is approximately the same as the slab.

XUV images at $256 \mathrm{eV}$ were obtained for targets with and without $\mathrm{CH}$ cones attached [see Figs. 3(c) and 3(a), respectively]. When plane slabs were irradiated both at 0 and at $43^{\circ}$ there was a clear ring structure visible in the heated region on the rear surface. The full width half maximum (FWHM) of a lineout of intensity through the center of the image in the vertical direction was an average of $65 \pm 10 \mu \mathrm{m}$. When the cone slabs were irradiated the ring structure disappeared and instead the arbitrary intensity peaked on axis with an average FWHM of $44 \pm 10 \mu \mathrm{m}$. The average peak counts (which is proportional to maximum temperature [12]) for the slab with no $\mathrm{CH}$ cone was $2 \times 10^{5}$ and with $\mathrm{CH}$ cone 2.5 $\times 10^{5}$, which is not a significant difference.

The lateral decrease in size seen in the shadowgrams is in agreement with the XUV data. Since the peak counts in the slab and the cone-slab case are similar, this agrees well with the similar longitudinal extent of the expansion in both cases observed in the shadowgraphy.

X-ray emission spectra were obtained for these shots. An example is shown in Fig. 4. The measured $\mathrm{X}$-ray spectra show Ly- $\alpha$, He- $\alpha$, He- $\beta$, and He- $\gamma \mathrm{Al}$ resonance emission lines. The spectra were analyzed using a model that combines collisional-radiative atomic kinetics with spectroscopic quality radiation transport and Stark line-broadening effects 


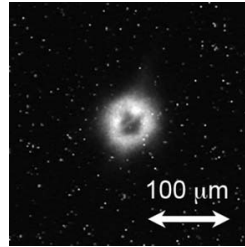

(a)

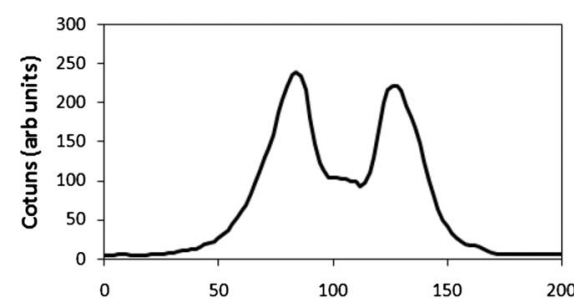

(b)

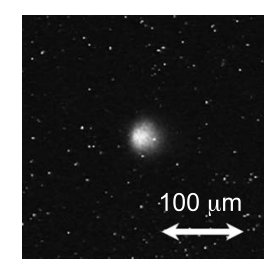

(c)

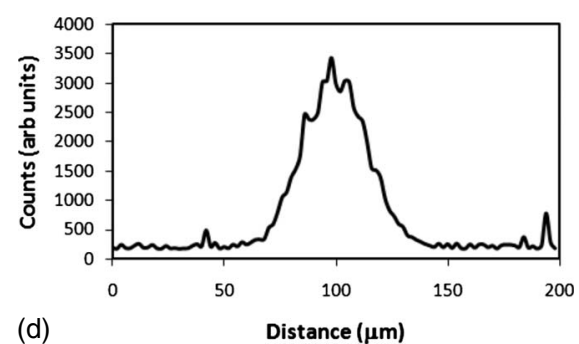

FIG. 3. XUV images of $4 \mu \mathrm{m} / 0.2 \mu \mathrm{m} / 4 \mu \mathrm{m} \mathrm{CH} / \mathrm{Al} / \mathrm{CH}$ slab targets without (a) and with (c) a plastic guide cone attached. Figures (b) and (c) are the lineouts taken from the images.

(for details see Ref. [13]). By calculating synthetic spectra for single temperature and electron density and comparing with experimental data it was possible to estimate typical values of plasma conditions present during the observed line emission. Previous work shows that time integrated measurements can provide similar temperature information to time resolved [14].

The calculated spectra were normalized to the He- $\alpha$ resonance line and temperature and density were chosen to match the intensity of the Ly- $\alpha$ line. Based on this analysis, it was possible to infer characteristic temperature and density conditions of $610 \pm 50 \mathrm{eV}$ and $1 \times 10^{24} \mathrm{el} / \mathrm{cc}$ with the cone attached to the target and $790 \pm 50 \mathrm{eV}$ and $7 \times 10^{23} \mathrm{el} / \mathrm{cc}$ without the cone. In these calculations it was not possible to simultaneously reproduce the satellite emission lines on the red wings of the He- $\alpha$ and Ly- $\alpha$ lines. This can be attributed to the fact that the measured spectra are space and time integrated. The $\mathrm{He}-\beta$ and $\mathrm{He}-\gamma$ line were also not reproduced using this method alone. More detailed atomic physics calculations were required to reproduce these lines and are not discussed here. This will be the subject of a future publication. The difference in temperature between the two target types could arise due to the fact that there is simply more material to heat in the case of the reentrant cone geometry. Having more material also changes refluxing of fast electrons and therefore the heating of the target. In thin targets electrons can make many passes before the end of the laser pulse. However, the reentrant cone geometry has a large

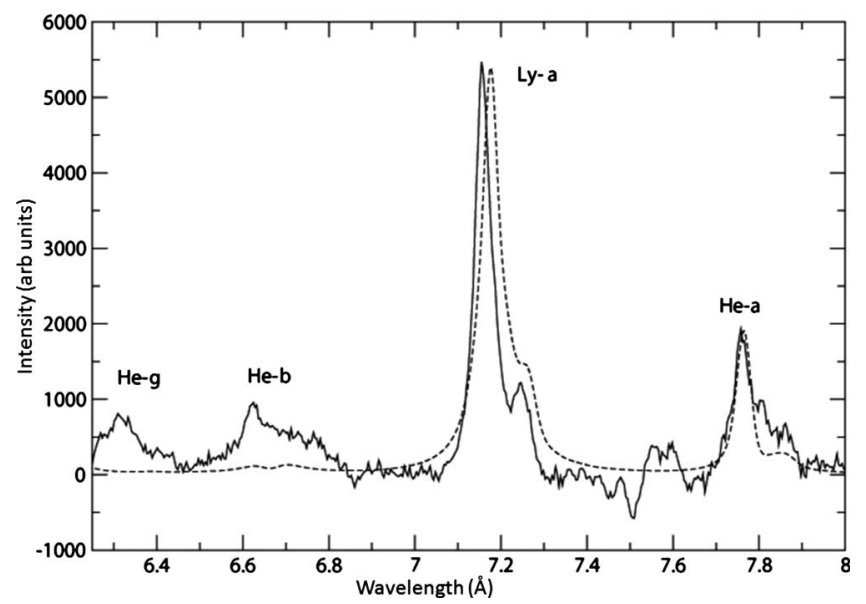

FIG. 4. Comparison of measured (solid line) and synthetic (dotted line) spectra for the target without a guiding cone $T=790 \mathrm{eV}$ and $N_{e}=7 \times 10^{23} \mathrm{el} . / \mathrm{cc}$. amount of material either side of the interaction region which would prevent refluxing.

The unusual ring structure observed in Fig. 3(a) is unlikely to be the beam hollowing [15]. This is because the rapid rise in temperature reduces the resistivity. In the case of resistivity decreasing with temperature the hollowing effect is counteracted. A similar ring structure has been observed in soft x-ray images of buried layer targets previously published by Koch et al. [16]. From their detailed discussion, they suggest that it is a Weibel-like instability driven development of an annular pattern. This could be on explanation for the ring structure observed here in this work. The transport pattern behavior with and without cone geometry was repeatable for all shots using such targets. The Annular/ring structures were consistent both within this experiment and on subsequent experiments using the same laser with similar targets.

In order to assess the effect of refluxing electrons on the heating pattern on the target's rear surface we have constructed a simple 2D model which assumes, for simplicity, that the laser-produced fast electrons propagate out of the absorption region in a conical beam. We assume that the beam propagates ballistically out of the absorption region and is specularly reflected at the rear surface. The reflected backward propagating beam can then be allowed to specularly reflect at the target front surface and propagate back through the target with a reduced current density. This process may be repeated an arbitrary number of times to include the effect of multiple beam refluxing.

Each successive ( $N$ th) beam can be thought of as propagating from the origin of its own spherical coordinate system $\left(r_{N}, \theta_{N}\right)$, with $\theta$ as the polar angle and azimuthal symmetry is assumed. If $\phi$ is the beam divergence angle, then the current density of the $N$ th beam will be $\mathbf{j}=\mathbf{j}_{\mathbf{r}_{\mathbf{N}}} \hat{\mathbf{r}}_{\mathbf{N}}$ :

$$
j_{r_{N}}=\frac{j_{0}}{J_{N}}\left(\frac{R}{r_{N}}\right)^{2} U\left(\phi-\theta_{N}\right),
$$

where $U$ is the unit step function and $J_{N}$ is a normalization integral. $R$ is the distance from the beam focal point to the beam edge on the front surface.

The total current density along the rear surface $j_{y}$ $=2 j \sin \theta$ can be found by summing the above equation over $N$. The factor of 2 in the expression for $j_{y}$ arises because the transverse components of the reflected and incident electron fluxes are in the same direction (while the longitudinal fluxes are antiparallel and therefore cancel at the rear surface). 
If we next assume that the dominant heating mechanism of the background electrons is joule heating by the resistively-induced electric field due to the net beam current, then the temperature on the rear surface will be given by the solution to the simple energy equation $n_{e} \partial T_{e} / \partial t=-\mathbf{j} \cdot \mathbf{E}$, where $n_{e}$ is the electron density, $T_{e}$ is the electron temperature, and $\mathbf{E}=\eta\left(\mathbf{T}_{\mathbf{e}}\right) \mathbf{j}$, with $\eta\left(T_{e}\right)$ as the resistivity. Since the target is initially cold, Spitzer resistivity does not apply and we use the hybrid resistivity described in [17] to obtain a numerical solution to the above equation. It was assumed that: the initial beam radius was $5 \mu \mathrm{m}$; the beam divergence angle was $60^{\circ}$; the current density at the target front surface due to laser-accelerated fast electrons was $=4 \times 10^{16} \mathrm{~A} \mathrm{~m}^{-2}$; the beam duration was $500 \mathrm{fs}$ and the target was composed of 8 - $\mu \mathrm{m}$-thick solid $\mathrm{CH}$ with an average ionization of $Z$ =3.5. The solution is plotted in Fig. 5, which shows two cases: the first assumes the beam undergoes ten successive forward-and-backward passes through the target, which represents the case of a thin isolated target; the second assumes only a single forward-and-backward pass, which represents the cone target, which is able to absorb any electrons passing back to the front surface. The two different heating patterns in Fig. 5 match aspects of the experimental observations: the cone target has a somewhat lower peak temperature and limited radial extent, while the isolated target shows a higher peak temperature and significantly wider radial extent because the multiple refluxing leads to more heating over an extended area. Thus, this model suggests that the reason for the reduced temperature and radial extent of the heating pattern on the rear surface of cone targets is due to the absorption by the cone material of refluxing fast electrons, which prevents multiple refluxes. This situation is much closer to the transport physics of fast ignition cone-shell implosions. In conclusion, for these specific target geometries the data can be explained completely by changes in refluxing of the fast electron beam. Despite the fact that the energy of the

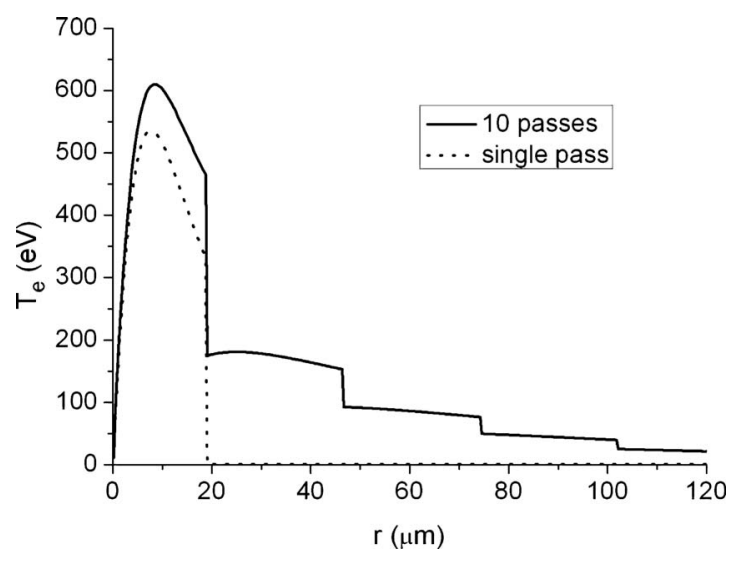

FIG. 5. The electron temperature on the rear surface as a function of the transverse distance from the target axis calculated according to the analytic model described in the text. The dashed line is the result for a single forward- and-backward pass through the target, while the solid line is for 10 such passes.

laser was contained within a $60 \mu \mathrm{m}$ spot (including the wings) and the tip of the cone was $30 \mu \mathrm{m}$ (conditions conducive to guiding according to theory), there was no reason to invoke guiding of the electron beam due to self-generated magnetic and electric fields.

This work was supported by the UK Engineering and Physical Sciences Research Council and the Science and Technology Facilities Council. American colleagues acknowledge support from the (U.S.) Department of Energy Contracts No. W-7405-Eng-48 and No. DE-FC5208NA28302. Japanese colleagues acknowledge the Japan Society for the Promotion of Science. The authors gratefully acknowledge the support of the staff of the Central Laser Facility and Dr. David Hoarty (AWE plc) for his assistance with this work.
[1] R. Kodama et al., Nature (London) 432, 1005 (2004).

[2] J. S. Green et al., Nat. Phys. 3, 853 (2007).

[3] R. Kodama et al., Nature (London) 412, 798 (2001).

[4] Y. Sentoku et al., Phys. Plasmas 11, 3083 (2004).

[5] M. H. Key et al., IFSA 2003 Proceedings, (American Nuclear Society, La Grange Park, IL,2004), Vols. 353-357.

[6] R. Kodama et al., Fusion Sci. Technol. 49. 316 (2006).

[7] C. N. Danson et al., Nucl. Fusion 44, S239 (2004).

[8] P. K. Patel et al., Plasma Phys. Controlled Fusion 47, B833 (2005).
[9] I. O. Musgrave et al., Appl. Opt. 46, 6978 (2007).

[10] T. Hall, J. Phys. E 17, 110 (1984).

[11] J. Howe et al., Rev. Sci. Instrum. 77, 036105 (2006).

[12] P. Gu et al., Rev. Sci. Instrum. 77, 113101 (2006).

[13] U. Andiel et al., Europhys. Lett. 60, 861 (2002).

[14] R. G. Evans et al., Appl. Phys. Lett. 86, 191505 (2005).

[15] J. R. Davies, Phys. Rev. E 68, 056404 (2003).

[16] J. A. Koch et al., Phys. Rev. E 65, 016410 (2001).

[17] J. R. Davies, A. R. Bell, M. G. Haines, and S. M. Guerin, Phys. Rev. E 56, 7193 (1997) 